\title{
Writing on Fading Paper* and Causal Transmitter CSI
}

\author{
Shashi Borade Lizhong Zheng \\ Laboratory for Information and Decision Systems \\ Massachusetts Institute of Technology \\ Cambridge, MA 02139, USA \\ $\{$ spb,lizhong\}@mit.edu
}

\begin{abstract}
A wideband fading channel is considered with causal channel state information (CSI) at the transmitter and no receiver CSI. A simple orthogonal code with energy detection rule at the receiver (similar to [6]) is shown to achieve the capacity of this channel in the limit of large bandwidth. This code transmits energy only when the channel gain is large enough. In this limit, this capacity without any receiver CSI is the same as the capacity with full receiver CSI-a phenomenon also true for dirty paper coding. For Rayleigh fading, this capacity (per unit time) is proportional to the logarithm of the bandwidth. Our coding scheme is motivated from the Gel'fand-Pinsker [2,3] coding and dirty paper coding [4]. Nonetheless, for our case, only causal CSI is required at the transmitter in contrast with dirty-paper coding and Gel'fand-Pinsker coding, where non-causal CSI is required.

Then we consider a general discrete channel with i.i.d. states. Each input has an associated cost and a zero cost input "0" exists. The channel state is assumed be to be known at the transmitter in a causal manner. Capacity per unit cost is found for this channel and a simple orthogonal code is shown to achieve this capacity. Later, a novel orthogonal coding scheme is proposed for the case of causal transmitter CSI and a condition for equivalence of capacity per unit cost for causal and noncausal transmitter CSI is derived. Finally, some connections are made to the case of non-causal transmitter CSI in [8].
\end{abstract}

\section{Introduction}

We consider a wireless fading channel of a large bandwidth $W$. The input $\mathbf{x}_{k}[i]$ of band $k$ at time $i$ is related to the output $\mathbf{y}_{k}[i]$ as:

$$
\mathbf{y}_{k}[i]=\mathbf{h}_{k}[i] \mathbf{x}_{k}[i]+\mathbf{n}_{k}[i] \quad 1 \leq k \leq W, \quad i \in\{1,2,3 \cdots\}
$$

where $\mathbf{n}_{k}[i]$ is complex circularly symmetric white Gaussian noise of unit variance. Each $\mathbf{n}_{k}[i]$ is independent of all inputs, fading gains, and noise in other bands. The fading

*These results were first mentioned briefly in Allerton Conference, October 2004 and later in LIDS Student Conference, January 2005. 
gains $\left\{\mathbf{h}_{k}[i]\right\}$ are complex Gaussian with variance 1 and are assumed i.i.d. over time and frequency. The transmitter has an average power constraint at any time $i$ :

$$
\sum_{k=1}^{W} \mathcal{E}\left[\left|\mathbf{x}_{k}[i]\right|^{2}\right] \leq P \quad \forall i
$$

Note that the channel state at time $i$ is completely described by the $W$ channel gains $\left\{\mathbf{h}_{k}[i]: 1 \leq k \leq W\right\}$. We assume (for reasons discussed later) that at each time $i$, the transmitter knows this state, i.e. all $W$ fading gains at that time and the receiver has no such knowledge. That we are assuming full transmitter CSI and no receiver CSI.

The case of causal transmitter CSI and no receiver CSI was studied by Shannon for discrete channels [1]. A discrete channel having $|\mathcal{X}|$ possible inputs and $|\mathcal{S}|$ possible states (varying in i.i.d. manner), can be converted to a discrete memoryless channel of same output alphabet but a larger input alphabet of size $|\mathcal{X}|^{|\mathcal{S}|}$. Capacity of the original channel equals that of this memoryless channel, which is easier to analyze.

Later, 2, 3] studied the following modification of this scenario. There the channel state for the entire codeword is known to the transmitter before beginning its transmission. Thus the CSI is available to the transmitter in a non-causal manner, whereas the receiver has no CSI at all. The optimal code in this case has a large number of candidate codewords for each message. The candidate which is suitable to the entire state-sequence spanning the code-length is used for transmission. More precisely, a candidate which is jointly typical with the state-sequence is used for transmission. This motivates our coding scheme for this wideband fading channel, where the codeword candidate which benefits the most from the state sequence is used for transmission.

For the wideband fading channel above, the capacity without any receiver and transmitter CSI can be achieved by an orthogonal coding scheme like Pulse-Position Modulation or Frequency-Shift Keying [5, 6]. In the limit of large bandwidth, this capacity without any CSI equals the capacity with full receiver CSI, which is $P \log _{2} e$ bits per unit time.

For the case of full CSI at both ends, the capacity is achieved by water-filling which transmits power only when the channel gain is large enough and this capacity was shown to be essentially $P \log _{2} W$ bits per unit time for the Rayleigh fading case [7]. For the intermediate case of only transmitter CSI, we wish to combine these two ideas of orthogonal coding and water-filling. We show that one can combine these two ideas without loss of optimality, that is, a code combining these two ideas is shown to achieve the capacity of this channel. This capacity with only transmitter CSI turns out to be essentially the same as the capacity $\left(\approx P \log _{2} W\right.$ bits per unit time) with both transmitter and receiver having CSI. This is another example where receiver CSI (or lack of it) does not affect the wideband capacity. In fact, it turns out that this capacity can be achieved by the proposed code with only one bit of transmitter CSI for each channel gain without any receiver CSI.

After noting that transmitter CSI can significantly (by a factor of $\ln W$ ) increase the capacity of a wideband fading channel irrespective of receiver CSI, we address the assumption of having transmitter CSI without any receiver CSI. This may seem to be a peculiar assumption for a wireless system because the transmitter in a typical wireless system obtains its CSI through feedback from the receiver itself. Nonetheless, after feeding back CSI to the transmitter, the receiver may want to ignore the CSI for multiple reasons-especially since this does not hurt capacity. 
- Ignoring CSI at the receiver may help in simplifying the decoding algorithm. The structure of the proposed orthogonal code (for a receiver with no CSI) may simplify the decoder.

- Another reason for ignoring receiver CSI comes from the fact that obtaining CSI at the receiver is intrinsically costly (e.g. in terms of energy spent in training for CSI). We see later (in section 2) that if receiver CSI is ignored, obtaining CSI for all channels is not necessary. CSI needs to be obtained only for a small fraction of channels which reduces the overall cost of obtaining CSI. This saving in the channel estimation cost (energy) can bring significant gains in this wideband system, where the available energy per degree of freedom is severely limited.

- In addition to less frequent CSI estimation, ignoring receiver CSI allows for a coarse channel estimation. As discussed in section 2, the proposed orthogonal code for a receiver with no CSI requires only one bit of CSI per channel. Obtaining this single bit of CSI might be easier compared to estimating the exact channel gain.

The next section describes the coding scheme and proves its achievable rate. Section 3 considers a general discrete channel with states. For the case of causal CSI, an achievable rate for this channel is proved with an orthogonal code. This is later shown to equal its capacity. In the last section, the case of causal transmitter CSI is used to interpret the case of non-causal transmitter CSI [8].

\section{Capacity achieving scheme}

Our coding scheme is a modification of a scheme like Frequency-Shift Keying scheme or Pulse-Position Modulation, that is, here the transmitter only transmits if the fading gain is large. The purpose here is to exploit the channel randomness instead of combating it. We will split the total bandwidth $W$ into $K$ pieces, each of bandwidth $w=W / K$ and these pieces would be used separately for communication. The available power $P$ is also equally divided into these pieces. Next, we illustrate our coding scheme for one such piece and analyze its achievable rate $r$. The total achievable rate would be number of pieces $K$ times the rate per piece $r$. We will use the notation $f(x) \approx g(x)$ to denote $\lim _{x \rightarrow \infty} \frac{f(x)}{g(x)}=1$.

The code for such a piece of bandwidth $w$ spans $T$ symbols in time. This code uses each of the $T$ time indices to denote a message from the set $\{1,2 \cdots T\}$. Thus $\ln T$ information nats ${ }^{1}$ are transmitted in time $T$ and hence the code rate is $\ln T / T$ nats per unit time. Say a total of $\lambda$ energy units are available for this. When message $j$ is to be transmitted, these $\lambda$ energy units will be transmitted only at time $j$. Moreover, these entire $\lambda$ units of energy are transmitted on a single frequency band say $f_{j}$ (see Figure 11). This is the first band where the channel gain for time $j$ is larger than a threshold $\Phi=\ln w-\ln (2 \ln w)$.

$$
f_{j}=\min \left\{i:\left|\mathbf{h}_{i}[j]\right|^{2} \geq \Phi\right\}
$$

Note that causal transmitter CSI is enough for this purpose. A type I error is declared if the channel gains at the time of message $j$ do not cross this threshold for any band.

\footnotetext{
${ }^{1} \ln 2$ nats $=1$ bit. Hence $\ln T$ nats equals $\log _{2} T$ bits. Unless mentioned otherwise, units of rate are nats per unit time.
} 
The decoder calculates the average (over $w$ bands) received energy $E_{i}$ for each time index $i$

$$
E_{i}=\frac{1}{w} \sum_{k=1}^{w}\left|\mathbf{y}_{k}[i]\right|^{2} \quad 1 \leq i \leq T
$$

The time index for which $E_{i}$ is maximum is declared as the transmitted message. Note that no channel state information is needed for this decoding method.

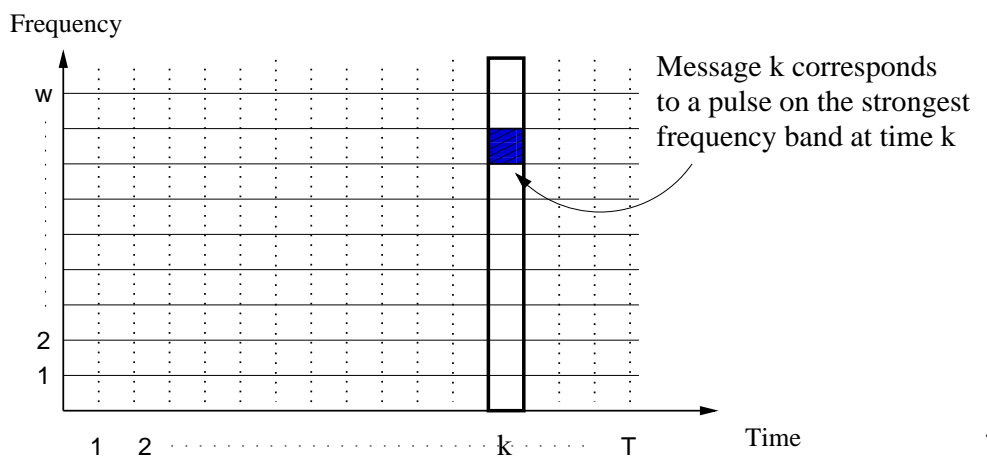

Figure 1: Proposed coding scheme: colored symbol indicates energy transmitted.

Without loss of generality, we will assume that message 1 was transmitted. A type II error is declared if $E_{i}$ the largest for some time other than the time of message 1. First, we show that probability $P_{I}$ of type I error goes to zero for large $w$. Note that type I error occurs if and only if the channel gains of all $w$ bands at time 1 are smaller than $\Phi$, that is, the maximum of those $w$ channel gains is smaller than $\Phi$. Since each $\left|\mathbf{h}_{k}[1]\right|^{2}$ is exponentially distributed, in the limit of large $w$, their maximum converges in distribution to [10]:

$$
\ln w+\mathbf{z} ; \text { where distribution of } \mathbf{z} \text { is } P(\mathbf{z} \leq Z)=\exp \left(-e^{-Z}\right)
$$

With our choice of the threshold $\Phi$, probability of type I error is

$$
P_{I}=P(\mathbf{z} \leq-\ln (2 \ln w))=1 / w^{2}
$$

Thus probability of type I error vanishes as $w$ tends to infinity. Now we show that probability $P_{I I}$ of type II error also vanishes as $w$ tends to infinity. Assuming that at time 1, the channel gain of band $i$ crosses the threshold $\Phi$ (i.e. $f_{1}=i$ ), the received symbol in band $i$ at time 1 is

$$
\begin{aligned}
\mathbf{y}_{i}[1] & =\mathbf{h}_{i}[1] \sqrt{\lambda}+\mathbf{n}_{i}[1] \\
\Rightarrow\left|\mathbf{y}_{i}[1]\right|^{2} & =\left|\mathbf{h}_{i}[1]\right|^{2} \lambda+\left|\mathbf{n}_{i}[1]\right|^{2}+2 \sqrt{\lambda} \Re\left(\mathbf{h}_{i}[1] \cdot \mathbf{n}_{i}[1]^{*}\right) \\
& \geq \Phi \lambda+\left|\mathbf{n}_{i}[1]\right|^{2}+2 \sqrt{\lambda} \Re\left(\mathbf{h}_{i}[1] \cdot \mathbf{n}_{i}[1]^{*}\right)
\end{aligned}
$$

Using Eq. (3), for large $w$

$$
\begin{aligned}
P\left(\left|\mathbf{h}_{j}[1]\right|^{2} \geq \ln w+2 \ln w\right) & =1-\exp \left(-\frac{1}{w^{2}}\right) \\
& \approx \frac{1}{w^{2}} \stackrel{w \rightarrow \infty}{\rightarrow} 0
\end{aligned}
$$

Now assuming $\left|\mathbf{h}_{j}[1]\right|^{2} \leq 3 \ln w$ yields

$$
\Re\left(\mathbf{h}_{j}[1] \cdot \mathbf{n}_{j}[1]^{*}\right) \geq-\left|\mathbf{h}_{j}[1]\right|\left|\mathbf{n}_{j}[1]\right| \geq-\sqrt{3 \ln w}\left|\mathbf{n}_{j}[1]\right|
$$


Note that $\left|\mathbf{n}_{j}[1]\right|^{2}$ is an exponential random variable with mean 1 . Hence

$$
P\left(\left|\mathbf{n}_{j}[1]\right|^{2} \geq 2 \ln w\right)=1 / w^{2}
$$

Now assuming $\left|\mathbf{n}_{j}[1]\right|^{2} \leq 2 \ln w$ implies

$$
\begin{aligned}
\Re\left(\mathbf{h}_{j}[1] \cdot \mathbf{n}_{j}[1]^{*}\right) & \geq-\sqrt{3 \ln w} \sqrt{2 \ln w} \\
\text { Substituting in (6) implies } \quad\left|\mathbf{y}_{j}[1]\right|^{2} & \geq \Phi \lambda-2 \sqrt{6 \lambda} \ln w+\left|\mathbf{n}_{i}[1]\right|^{2}
\end{aligned}
$$

The above statement may fail if either of the events in Eq. (41),(9), (10) occurs. By union bound, this probability is at most $3 / w^{2}$. Also note that the noise energy equals the received energy in all other bands where no energy is transmitted. Hence with at least a probability of $1-3 / w^{2}$, the average received energy at time 1 follows:

$$
\begin{aligned}
E_{1} & \geq \frac{\Phi \lambda-2 \sqrt{6 \lambda} \ln w}{w}+\frac{\sum_{k=1}^{w}\left|\mathbf{n}_{k}[1]\right|^{2}}{w} \\
& \triangleq \alpha+\frac{\sum_{k=1}^{w}\left|\mathbf{n}_{k}[1]\right|^{2}}{w}
\end{aligned}
$$

where $\alpha$ equals the first term in (11) and is a non-random variable. By weak law of large numbers, the second term in (11) converges to 1 for large $w$. Average received energy $E_{t}$ at any other time $t \neq 1$ equals

$$
E_{t}=\frac{\sum_{k=1}^{w}\left|\mathbf{n}_{k}[t]\right|^{2}}{w}
$$

A type II error occurs when any of these $E_{t}$ exceeds $\alpha+1$. Since each $\left|\mathbf{n}_{k}[t]\right|^{2}$ is an exponential random variable with mean 1, applying Chernoff's bound on similar lines of [6] gives

$$
\begin{aligned}
P\left(E_{t} \geq \alpha+1\right) & \leq \exp (-w L(\alpha)) \\
\text { where } L(\alpha) & =\alpha-\ln (\alpha+1)
\end{aligned}
$$

Applying union bound over all wrong messages from 2 to $T$, we get the following bound on type II error probability

$$
P_{I I} \leq T \exp (-w L(\alpha))=\exp (-w(L(\alpha)-\ln T / w))
$$

Since $P_{I}$ goes to zero with increasing $w$ as shown before, the overall error probability vanishes with increasing $w$ if $P_{I I}$ also vanishes with increasing $w$. This happens if

$$
\begin{aligned}
\ln T / w & <L(\alpha) \\
\Rightarrow \quad \ln T / T & <\frac{w}{T} L(\alpha)=\frac{w}{T}(\alpha-\ln (\alpha+1))
\end{aligned}
$$

Thus the maximum achievable rate $^{2}$ depends on $\alpha$ and hence depends on $\lambda$ (because $\alpha$ equals $\left.\frac{\Phi \lambda-2 \sqrt{6 \lambda} \ln w}{w}\right)$.

\footnotetext{
${ }^{2}$ We have shown that the error probability of this orthogonal code goes to zero as $w$ goes to infinity. However, a subtle point is that for showing a rate $\ln T / T$ is achievable, we have to show that arbitrarily small error probability can be achieved for a given (but large) $w$ and $T$. As shown in [12, this can be achieved by coding over many blocks of our orthogonal code by treating the orthogonal code as the inner code of this concatenated code. The orthogonal code provides an essentially noiseless discrete memoryless channel (with input cardinality $T$ ) for the outer code. Thus a rate $\approx \ln T / T$ can be achieved.
} 
Let this scheme be applied only for $\delta$ fraction of the time where $\delta$ is a suitably chosen parameter. No communication happens in the remaining fraction of time. Thus if $p$ is the overall average power available for this piece of bandwidth, $p / \delta$ is the average power available when communication is being done. Thus the peakiness denoted by $\delta$ boosts the power level for actual communication by a factor of $1 / \delta$. This boost in power level is necessary for the success of this orthogonal code. It ensures that the energy pulse transmitted (for the correct message) is strong enough to be identifiable at the receiver from incorrect messages.

Since the time-length of this code is $T$, total transmit energy $\lambda$ for this code is equal to $T p / \delta$. Since communication happens for only $\delta$ fraction of time, the overall maximum achievable rate is given by

$$
r=\delta \frac{w}{T} L\left(\alpha^{*}\right) \quad \text { where } \quad \alpha^{*}=\frac{\Phi p T / \delta-2 \sqrt{6 p T / \delta} \ln w}{w}
$$

Since the total available power $P$ is divided equally amongst $K$ pieces of the total bandwidth, power available per piece equals $p=P / K$. We choose $K=\ln w$ and $\delta=\epsilon T / w$, where $\epsilon>0$ is a small number. Substituting these values yields $\alpha^{*} \approx P / \epsilon$. Now note that $L(\alpha) \approx \alpha$ for large $\alpha$. Since $\alpha^{*}$ can be made arbitrarily large by choosing small enough $\epsilon$, the maximum achievable rate is given by

$$
\begin{aligned}
r & =\delta \frac{w}{T} L\left(\alpha^{*}\right) \approx \delta \frac{w}{T} \alpha^{*} \\
& \approx \frac{\epsilon T}{w} \frac{w}{T} \frac{P}{\epsilon}(=P)
\end{aligned}
$$

Since there are $K=\ln w$ such pieces of bandwidth $w$, the total rate $r K$ equals $P \ln w$ nats per unit time. The total bandwidth for these $K$ pieces equals $W=w \ln w$. Noting that $\ln W \approx \ln w$, the total rate is given by $R \approx P \ln W$ nats per unit time.

This rate expression matches the capacity of this fading channel when the receiver and transmitter both have full CSI [7. This proves that the proposed coding scheme achieves the capacity for this channel with no receiver CSI. Thus the lack of receiver CSI does not reduce capacity-a phenomenon similar to writing on dirty paper.

Theorem 1 Capacity of the Rayleigh fading wideband channel with causal transmitted CSI and no receiver CSI is achieved by the proposed coding scheme. In the limit of large bandwidth, this capacity $C \approx P \ln W$ nats per unit time and is unchanged if even the receiver has full CSI.

Note that as mentioned in Section 1, full transmitter CSI is not needed for the proposed scheme; only one bit of CSI is enough for each channel gain $\mathbf{h}_{i}[j]$. This bit indicates whether or not the channel gain is above the threshold $\Phi$. Also note that CSI is not needed at every time for this scheme. Since there is no activity for $(1-\delta)$ fraction of time and only $\delta$ fraction of the time is used for communication, only this $\delta$ fraction of time needs CSI and hence the cost of obtaining CSI is significantly reduced. Since the capacity of a wideband channel with full receiver and transmitter CSI (at all times) is essentially the same as the capacity of our channel with only transmitter CSI (for only a fraction $\delta$ of time), one may want to mimic no receiver CSI even when it is available!

We can extend above analysis for the case of noisy transmitter CSI, where the channel gain $\mathbf{h}_{i}[j]$ equals the sum of two independent complex Gaussian components, $\mathbf{g}_{i}[j]$ and $\mathbf{f}_{i}[j]$, which are i.i.d. over frequency and time. Transmitter only knows $\left\{\mathbf{g}_{i}[j]\right\}$ and the 
error $\mathbf{f}_{i}[j]$ is independent of $\mathbf{g}_{i}[j]$. The variance of the known component is $\beta \in(0,1]$ and hence that of the error is $1-\beta$. A code similar to the perfect CSI case is employed. For example, if message 1 is to be transmitted, the transmitter transmits energy only in the frequency band where the known channel strength $\left|\mathbf{g}_{i}[1]\right|^{2}$ is larger than $\beta \Phi$. Thus the threshold for the perfect transmitter CSI case is reduced by a factor of $\beta$. This scheme can be shown to achieve a rate of $\beta P \ln W$ nats per unit time. This again equals the capacity when receiver also has full CSI [7]. Thus again receiver CSI is irrelevant for capacity in the limit of large bandwidth.

Remark 1: Similar results can be proved when distribution of the fading gain $\left|\mathbf{h}_{i}[j]\right|^{2}$ is not exactly exponential but has an exponential tail. If the tail behaves similar to an exponential with mean $m$, the capacity can be shown to be $m P \ln W$ nats per unit time.

Remark 2: Similar analysis can be performed if the tail of the fading gain distribution is a polynomial, that is, $P\left(\left|\mathbf{h}_{i}[j]\right|^{2} \geq x\right) \approx x^{-n}$ for some $n>0$. In that case, the proposed code achieves a rate $R \approx P W^{\frac{1}{n+1}}$ nats per unit time. This again turns out to be the same as the capacity when the receiver also has full CSI.

Finally, (on similar lines of [8]) we can interpret the proposed scheme in terms of the binning argument in [2, 3]. For the binning interpretation, logarithm of the number of codewords per message should equal the mutual information between the state sequence $\left\{\mathbf{h}_{j}[i]\right\}$ and the input sequence $\left\{\mathbf{x}_{j}[i]\right\}$. The number of possible codewords per message equals $w$ in our code as energy can be transmitted on any of the $w$ bands available in a piece. Now note that in our code, the state sequence completely determines the input sequence for a given message, because we transmit all energy only where the channel gain first crosses the threshold $\Phi$. Hence the above mutual information equals the input entropy for a message. Since probability of no frequency band crossing the threshold goes to zero for large $w$ and any of the $w$ frequency bands are equally likely to cross the threshold, entropy of the input tends to $\log w$. Thus the binning interpretation is justified as the logarithm of the number of possible codewords per message equals the mutual information between the input and state sequences.

\section{Capacity per cost with causal transmitter CSI}

We saw in the previous section how the proposed orthogonal code achieved the capacity of the wide-band fading channel with no receiver CSI. It also means that the proposed code achieved the capacity per unit cost for that channel. This section analyzes the case of causal transmitter CSI for a more general channel.

The random variables at time $i \in\{1,2,3 \cdots\}$ corresponding to the channel input $X_{i}$, output $Y_{i}$ and channel state $S_{i}$ take values from the sets $\mathcal{X}, \mathcal{Y}$ and $\mathcal{S}$ respectively ${ }^{3}$. State $S$ defines a channel transition matrix denoted by $P_{Y \mid X S}$. The states are assumed to change i.i.d. over time, that is, if $P_{S}(\cdot)$ denotes the distribution of $S_{i}$ then the probability of a state-sequence $s_{1}^{l}$ equals $\prod_{i=1}^{l} P_{S}\left(S_{i}=s_{i}\right)$. Conditioned on the state sequence, the channel is assumed to be memoryless i.e.

$$
P\left(Y_{1}^{l} \mid X_{1}^{l}, S_{1}^{l}\right)=\prod_{i=1}^{l} P_{Y \mid X S}\left(y_{i} \mid x_{i}, s_{i}\right)
$$

\footnotetext{
${ }^{3}$ Unless stated otherwise, capital letters denote random variables and small letters denote their values. Notation $X_{1}^{l}$ is used as a shorthand for the sequence $X_{1} X_{2} \cdots X_{l}$.
} 
Each input $x \in \mathcal{X}$ incurs a cost $b(x) \in[0, \infty)$. A zero cost input is assumed to exist and denoted by " 0 ". In a code of length $l$, the codeword for message $j$ is denoted by the sequence $x_{1}^{l}(j)$. A length $l$ code having $M \in\{1,2 \cdots\}$ messages is denoted by a $(l, M, \nu, \epsilon)$ code if the average probability of error is at most $\epsilon$ and codeword for every message $j$ satisfies the total cost constraint

$$
\sum_{i=1}^{l} b\left(x_{i}(j)\right) \leq \nu \quad 0 \leq j<M
$$

The capacity per unit cost for this channel is defined as in 9 .

Definition 2 For a given $0 \leq \epsilon<1$, rate (in nats) per unit cost $R$ is said to be $\epsilon$ achievable if for all every $\gamma>0$, there exists a $\nu_{0}$ such that for all $\nu \geq \nu_{0}, a(l, M, \nu, \epsilon)$ code can be found with $\ln M \geq \nu(R-\gamma)$. Rate per unit cost of $R$ is said to be achievable if $R$ is $\epsilon$-achievable for every $\epsilon>0$. Capacity per unit cost is the maximum achievable rate per unit cost.

We assume no receiver CSI and causal transmitter CSI, which means that the transmitter gets to know $S_{i}$ at time $i$ before transmitting $X_{i}$. Let $U: \mathcal{S} \rightarrow \mathcal{X}$ denote a mapping from states to inputs. This mapping $U$ is equivalent to a vector in $\mathcal{X}^{|\mathcal{S}|}$, where its each entry denotes the input mapped from the corresponding state. Let $P_{Y \mid U=u}(y)$ denote the output distribution induced when mapping $U=u$ is chosen, that is,

$$
P_{Y \mid U=u}(y)=\sum_{s \in \mathcal{S}} P_{S}(s) P_{Y \mid X S}(y \mid u(s), s)
$$

where $u(s)$ denotes mapping of state $s$ under $u$. We next prove the following theorem.

Theorem 3 Capacity per unit cost with no receiver CSI and causal transmitter CSI is given by

$$
\sup _{u} \frac{D\left(P_{Y \mid U=u}|| P_{Y \mid U=0}\right)}{\mathcal{E}[b(X) \mid U=u]}
$$

where $D\left(P_{Y \mid U=u}|| P_{Y \mid U=0}\right)$ denotes the relative entropy (in nats) between the output distributions induced when mapping $u$ is chosen and when identically zero mapping is chosen. $\mathcal{E}[b(X) \mid U=u]$ denotes the average cost incurred when mapping $u$ is chosen.

$$
\mathcal{E}[b(X) \mid U=u]=\sum_{s \in \mathcal{S}} P_{S}(s) b(u(s))
$$

Proof: We first show an orthogonal coding scheme which achieves the above rate per unit cost. We use the shorthand $f(n) \doteq g(n)$ to denote $\lim _{n \rightarrow \infty} \frac{\ln f(n)}{\ln g(n)}=1$. Similarly, $f(n) \leq g(n)$ and $f(n) \dot{<} g(n)$ are defined.

Choose a mapping $u: \mathcal{S} \rightarrow \mathcal{X}$. Our code of $M$ messages spans $M n$ symbols. Each message corresponds to a non-overlapping interval of length $n$, that is, message $j \in$ $[0, M-1]$ corresponds to interval ${ }^{5}[j n+1, j n+n]$. If message $j$ is to be transmitted, " 0 " is transmitted at all times except interval $[j n+1, j n+n]$. During each time $i \in$ $[j n+1, j n+n]$, input $u\left(S_{i}\right)$ is transmitted. This requires only causal CSI at the encoder.

\footnotetext{
${ }^{4}$ We assume that relative entropy and mutual information are measured in nats i.e. with natural logarithm.

${ }^{5}$ This means the set of integers from $j n+1$ to $j n+n$.
} 
Assuming message $j$ was transmitted, the output distribution at each time in interval $[j n+1, j n+n]$ is given by $P_{Y \mid U=u}$. Outputs in all other intervals are distributed as $P_{Y \mid U=0}$. For each of the $M$ intervals of length $n$, the decoder finds the empirical output distribution of that interval. Let $P_{Y}^{k}$ denote this empirical distribution for interval $[k n+1, k n+n]$. The interval $k$ for which $D\left(P_{Y}^{k} \| P_{Y \mid U=0}\right)$ is larger than a threshold ${ }^{6} \Phi=D\left(P_{Y \mid U=u}|| P_{Y \mid U=0}\right)-\delta$ is declared as the transmitted message, where $\delta$ is a chosen small number. An error is declared when none or multiple such intervals exist.

First kind of error occurs if the divergence $D\left(P_{Y}^{j} \| P_{Y \mid U=0}\right)$ for the correct interval does not exceed the threshold $D\left(P_{Y \mid U=u}|| P_{Y \mid U=0}\right)-\delta$. By Sanov's theorem (e.g. [11]), this probability goes to zero exponentially fast in $n$. Hence this probability of error of first kind is smaller than $\epsilon / 3$ for all $n \geq n_{1}$ for some $n_{1}>0$.

The second kind of error occurs if the divergence $D\left(P_{Y}^{k}|| P_{Y \mid U=0}\right)$ for a wrong interval $k \neq j$ exceeds the threshold. Again applying Sanov's theorem implies

$$
P\left(D\left(P_{Y}^{k}|| P_{Y \mid U=0}\right)>\Phi\right) \doteq \exp (-n \Phi)
$$

By union bound, the probability $P_{I I}$ that any of the $M-1$ wrong intervals crosses this threshold is bounded by

$$
P_{I I} \leq M \exp (-n \Phi)
$$

If we choose $M=\exp (n(\Phi-\delta))$, probability $P_{I I}$ also goes to zero exponentially as $\exp (-n \delta)$. Thus the probability of error of second kind is smaller than $\epsilon / 3$ for all $n \geq n_{2}$ for some $n_{2}>0$.

By i.i.d. nature of the states and the law of large numbers, total cost for each message is smaller than $n\left(\mathcal{E}[b(X) \mid U=u]+\delta\right.$ ) with (at least) a probability of $1-\epsilon / 3$ if $n \geq n_{3}$ is chosen for some $n_{3}>0$.

Thus even if an error of third kind is declared if the total cost of the codeword exceeds the threshold, the total probability of any kind of error is less than $3(\epsilon / 3)$ for all $n \geq \max \left(n_{1}, n_{2}, n_{3}\right)$. Thus for any $\epsilon>0$ and $\gamma>0$, we can choose small enough $\delta$ such that

$$
\begin{aligned}
\ln M=n(\Phi-\delta) & \\
& =n\left(D\left(P_{Y \mid U=u}|| P_{Y \mid U=0}\right)-2 \delta\right) \\
& >n(\mathcal{E}[b(X) \mid U=u]+\delta)\left(\frac{D\left(P_{Y \mid U=u}|| P_{Y \mid U=0}\right)}{\mathcal{E}[b(X) \mid U=u]}-\gamma\right)
\end{aligned}
$$

and the probability of error is smaller than $\epsilon$ for $n \geq \max \left(n_{1}, n_{2}, n_{3}\right) \triangleq n_{*}$. Substituting $\nu=n(\mathcal{E}[b(X) \mid U=u]+\delta)$ and $\nu_{0}=n_{*}(\mathcal{E}[b(X) \mid U=u]+\delta)$ in the definition of the rate per unit cost proves that the proposed orthogonal code achieves a rate per unit cost of $D\left(P_{Y \mid U=u}|| P_{Y \mid U=0}\right) / \mathcal{E}[b(X) \mid U=u]$.

Remark 3: Note the similarity of this scheme with the coding scheme in previous section for the wideband fading channel. In particular, note that the probability of an incorrect interval crossing the threshold $\Phi$ is given by $\exp (-n \Phi)$, similar to (14). For these reasons, one can interpret the divergence $D\left(P_{Y}^{i} \| P_{Y \mid U=0}\right)$ for interval $i$ as the discrete channel analogue of the average received energy $E_{i}$ for the wideband fading channel.

\footnotetext{
${ }^{6}$ This threshold is finite if the support of $P_{Y \mid U=u}$ is contained in that of $P_{Y \mid U=0}$. This threshold is not finite if there exists an output (say $\hat{y}$ ) which can only occur with a non-zero input. The decoding in that case would be easy because only the correct interval can have the output $\hat{y}$.
} 
Proof of converse: We first note the following upper bound in 9] on capacity per unit cost of a discrete memoryless channel with input $V$ and output $Z$

$$
\sup _{v} \frac{D\left(P_{Z \mid V=v}|| P_{Z \mid V=0}\right)}{c(v)}
$$

where $P_{Z \mid V=v}$ denotes the output transition probability for input $v, c(v)$ denotes the cost of input $v$ and $V=0$ denotes the zero cost input.

Now recall Shannon's idea [1] that this channel with causal transmitter CSI and i.i.d. states can be thought as a discrete memoryless channel (DMC) with the same output alphabet but a larger input alphabet. The input alphabet $U$ of that equivalent DMC corresponds to a mapping from $\mathcal{S}$ to $\mathcal{X}$ and thus its cardinality equals $|\mathcal{X}|^{|\mathcal{S}|}$. An input $U$ of this DMC is equivalent to a vector in $\mathcal{X}^{|\mathcal{S}|}$ made up of contingent inputs (from $\mathcal{X}$ ) for each state $s \in \mathcal{S}$. A code for the DMC can be converted to a code for causal transmitter CSI channel as follows. If the symbol $u_{i}$ was transmitted at time $i$ on the DMC, the transmitter with causal CSI transmits input $u_{i}\left(S_{i}\right)$ at time $i$ after observing state $S_{i}$.

This DMC is a cascade of two memoryless parts. First part chooses the state $S_{i}$ with distribution $P_{S}$ and picks the corresponding contingent input $u_{i}\left(S_{i}\right) \in \mathcal{X}$ from the transmitter. Second part is similar to our original channel of interest, which emits the output based on the state $S_{i}$ and the input $u_{i}\left(S_{i}\right)$ according to the distribution $P_{Y \mid X S}\left(\cdot \mid u_{i}\left(S_{i}\right), S_{i}\right)$. The output distribution of this DMC conditioned on the input $u$ is given by $P_{Y \mid U=u}$ in (18).

Finally, note that $\mathcal{E}[b(X) \mid U=u]$ denotes the (average) cost incurred due to choosing the DMC input $u$. The converse follows by applying (20) after replacing $P_{Z \mid V=v}$ by $P_{Y \mid U=u}$, $P_{Z \mid V=0}$ by $P_{Y \mid U=0}$ and $c(v)$ by $\mathcal{E}[b(X) \mid U=u]$. A more detailed converse is proved in the appendix.

We could also prove the direct part of this theorem using above method of conversion to a DMC. However, the earlier detailed proof is expected to be more insightful in view of writing on fading paper.

\section{Discussion}

For the wideband fading channel, we noted that the capacity with causal $\mathrm{CSIT}^{7}$ was the same as that with non-causal CSIT. Equivalently, the capacity per unit cost was the same with causal or non-causal CSIT. Similar phenomenon can be shown for the AWGN channel with additive Gaussian interference known at the transmitter by a modification of the scheme in [8]. We want to understand whether these are isolated examples (of the equivalence of capacity per unit cost with causal and non-causal CSIT) or they are special cases of a general class.

If with causal or non-causal CSIT, the capacities (for any given cost constraint) are the same for a channel; then it is easy to show that the capacities per unit cost would also be the same for that channel. This is because for a channel with a 0 cost alphabet, capacity per unit cost is given by the slope of the capacity vs. cost curve at 0 .

More interesting problem is to characterize the class of channels for which the capacity per unit cost is the same with causal or non-causal CSIT, but the capacity vs. cost curves are not the same for causal and non-causal CSIT. Above mentioned wideband AWGN channel and wideband fading channel with additive interference are two such channels.

\footnotetext{
${ }^{7}$ CSIT: Acronym for transmitter CSI.
} 


\subsection{Review of the non-causal transmitter CSI case}

We briefly summarize the coding scheme that achieves the capacity per unit cost with non-causal CSIT [8]. This code of $M$ messages spans $M q n$ symbols. Each message in this orthogonal code corresponds to a separate interval of length $q n$. For transmitting a message $j$, non-zero symbols can be only transmitted in the $j$ 'th interval of length $q n$. This message interval of length $q n$ can be thought as the set of $q$ subintervals, each of length $n$.

A distribution of states $\hat{P}_{S}(\cdot)$ is chosen beforehand. Out of these $q$ subintervals in the interval for message $j$, the subinterval whose empirical distribution is like $\hat{P}_{S}(\cdot)$ is chosen. More precisely, the divergence of the empirical distribution of this subinterval with respect to $\hat{P}_{S}(\cdot)$ should be small enough. Since the actual distribution of states is $P_{S}$, the probability of a subinterval having distribution like $\hat{P}_{S}(\cdot)$ is essentially (in $\doteq$ sense) given by $\exp \left(-n D\left(\hat{P}_{S} \| P_{S}\right)\right)$. We can find such a subinterval with high probability if the number of subintervals per message interval is

$$
q \doteq \exp \left(n D\left(\hat{P}_{S} \| P_{S}\right)\right)
$$

Non-zero symbols are only transmitted in this subinterval. A mapping $u: \mathcal{S} \rightarrow \mathcal{X}$ is also chosen beforehand. Similar to previous section, input $u(s)$ is transmitted for state $s$ in this subinterval. The output distribution in this subinterval would be

$$
\hat{P}_{Y}(y)=\sum_{s \in \mathcal{S}} \hat{P}_{S}(s) P_{Y \mid X S}(y \mid u(s), s)
$$

Output distribution in all other subintervals (where only input 0 is transmitted) is $P_{Y \mid U=0}$

$$
P_{Y \mid U=0}(y)=\sum_{s \in \mathcal{S}} P_{S}(s) P_{Y \mid X S}(y \mid 0, s)
$$

Note that non-zero symbols are transmitted in a small fraction $(1 / q)$ of the interval corresponding to message $j$. Note from (21) that this fraction decays exponentially to 0 with increasing $n$. Also note that non-causal CSIT is necessary to determine the subinterval having empirical distribution like $\hat{P}_{S}(\cdot)$.

At the receiver, empirical distribution is found for all the $q$ subintervals for each of the $M$ message intervals. If one of these $M q$ subintervals has distribution like $\hat{P}_{Y}$ in (22), the message interval containing that subinterval is declared as the transmitted message. An error is declared otherwise. Since every wrong subinterval is distributed as $P_{Y \mid U=0}$, probability of its having an empirical distribution as $\hat{P}_{Y}(y)$ is essentially $\exp \left(-n D\left(\hat{P}_{Y} \| P_{Y \mid U=0}\right)\right)$. Thus by union bound, the probability of a wrong subinterval having output distribution $\hat{P}_{Y}(y)$ is

$$
M q \exp \left(-n D\left(\hat{P}_{Y}|| P_{Y \mid U=0}\right)\right)=M \exp \left(n D\left(\hat{P}_{S}|| P_{S}\right)-n D\left(\hat{P}_{Y}|| P_{Y \mid U=0}\right)\right)
$$

Choosing $M \doteq \exp \left(n\left(D\left(\hat{P}_{Y} \| P_{Y \mid U=0}\right)-D\left(\hat{P}_{S} \| P_{S}\right)\right)\right)$ can ensure that probability error vanishes with large $n$. By law of large numbers, the total cost incurred for transmission is essentially $n \mathcal{E}_{\hat{P}_{S}}[b(u(S))]$ where

$$
\mathcal{E}_{\hat{P}_{S}}[b(u(S))]=\sum_{s \in \mathcal{S}} \hat{P}_{S}(s) b(u(s))
$$


Thus the rate per unit cost achieved by this scheme is

$$
\frac{\ln M}{n \mathcal{E}_{\hat{P}_{S}}[b(u(S))]} \doteq \frac{D\left(\hat{P}_{Y}|| P_{Y \mid U=0}\right)-D\left(\hat{P}_{S} \| P_{S}\right)}{\mathcal{E}_{\hat{P}_{S}}[b(u(S))]}
$$

Optimizing above expression over the choice of $\hat{P}_{S}$ and $u(\cdot)$ can be shown to yield the capacity per unit cost for this channel with non-causal CSIT. We denote an optimum choice by $\hat{P}_{S}^{*}$ and $u^{*}(\cdot)$, respectively.

\subsection{Adapting to the causal transmitter CSI case}

With causal CSIT, transmitter does not a priori know the subinterval having empirical state distribution $\hat{P}_{S}$. To overcome this issue, let there be only one subinterval per interval i.e. let $q=1$. Thus each message corresponds to an interval of length $n$. Now a fraction $\theta$ is chosen by the transmitter. The transmitter can only transmit energy (nonzero symbols) in a fraction $\theta$ of message interval. For each state $s \in \mathcal{S}$, the transmitter will transmit input $u(s)$ for the first $n \theta \hat{P}_{S}(s)$ occurrences of state $s$. Thus the states where energy is transmitted will have an empirical distribution $\hat{P}_{S}$. Since the actual state distribution is $P_{S}$, (by law of large numbers) an interval of length $n$ will have $n \theta \hat{P}_{S}(s)$ occurrences of state $s$ only if

$$
\theta \hat{P}_{S}(s) \leq P_{S}(s) \quad \forall s \in \mathcal{S} \quad \Rightarrow \theta \leq \inf _{s \in \mathcal{S}}\left\{P_{S}(s) / \hat{P}_{S}(s)\right\}
$$

Note that the $n \theta$ symbols where energy is transmitted need not be in a contiguous block. Again by law of large numbers, the total cost incurred in this procedure is (in $\approx$ sense) essentially $n \theta \mathcal{E}_{\hat{P}_{S}}[b(u(S))]$.

At the decoder, for each message interval of length $n$, the empirical output distribution is found for all $\left(\begin{array}{c}n \\ n \theta\end{array}\right)$ subsequences ${ }^{8}$ of length $n \theta$. Out of these $M\left(\begin{array}{c}n \\ n \theta\end{array}\right)$ subsequences, if all the subsequences having distribution like $\hat{P}_{Y}$ in (22) belong to a single message interval, the message corresponding to that message interval is declared as the transmitted message. An error is declared if more than one or none of the message intervals have such subsequences. By law of large numbers, the correct subsequence of length $n \theta$ where energy is transmitted will have an empirical output distribution like $\hat{P}_{Y}$ with high probability for large $n$. A length $n \theta$ subsequence in an incorrect message interval will have an empirical output distribution like $\hat{P}_{Y}$ with probability $p_{1}$ given by

$$
p_{1} \doteq \exp \left(-\theta n D\left(\hat{P}_{Y} \| P_{Y \mid U=0}\right)\right)
$$

Applying union bound, the probability of a subsequence of an incorrect message interval having empirical output distribution like $\hat{P}_{Y}$ is bounded by $M\left(\begin{array}{c}n \\ n \theta\end{array}\right) p_{1}$. Hence vanishing error probability can be achieved if

$$
\begin{aligned}
M \doteq \frac{1}{\left(\begin{array}{c}
n \\
n \theta
\end{array}\right) p_{1}} & =\frac{\exp \left(\theta n D\left(\hat{P}_{Y}|| P_{Y \mid U=0}\right)\right)}{\left(\begin{array}{c}
n \\
n \theta
\end{array}\right)} \\
& \doteq \frac{\exp \left(\theta n D\left(\hat{P}_{Y} \| P_{Y \mid U=0}\right)\right)}{\exp \left(n H_{b}(\theta)\right)} \quad \text { by Sterling Approximation } \\
& =\exp \left(n\left(\theta D\left(\hat{P}_{Y} \| P_{Y \mid U=0}\right)-H_{b}(\theta)\right)\right)
\end{aligned}
$$

\footnotetext{
${ }^{8}$ The term subinterval is reserved for contiguous blocks of symbols, whereas a subsequence need not be contiguous.
} 
The rate per unit cost achieved by this scheme equals

$$
\begin{aligned}
\frac{\ln M}{n \theta \mathcal{E}_{\hat{P}_{S}}[b(u(S))]} & =\frac{\theta D\left(\hat{P}_{Y} \| P_{Y \mid U=0}\right)-H_{b}(\theta)}{\theta \mathcal{E}_{\hat{P}_{S}}[b(u(S))]} \\
& =\frac{D\left(\hat{P}_{Y} \| P_{Y \mid U=0}\right)-H_{b}(\theta) / \theta}{\mathcal{E}_{\hat{P}_{S}}[b(u(S))]}
\end{aligned}
$$

For this to equal the capacity per unit cost with non-causal CSIT in (23), an optimum $\hat{P}_{S}^{*}$ maximizing (23) should satisfy

$$
H_{b}(\theta) / \theta=D\left(\hat{P}_{S}^{*} \| P_{S}\right)
$$

Now note that (24) implies

$$
\begin{aligned}
D\left(\hat{P}_{S}^{*} \| P_{S}\right) & =\sum_{s \in \mathcal{S}} \hat{P}_{S}^{*}(s) \ln \left(\frac{\hat{P}_{S}^{*}}{P_{S}}\right) \\
& \leq \sum_{s \in \mathcal{S}} \hat{P}_{S}^{*}(s) \ln \frac{1}{\theta}=\ln (1 / \theta) \\
& \leq H_{b}(\theta) / \theta
\end{aligned}
$$

Last step is met with equality either when $\theta=1$ or when $\theta$ tends to zero. For equality in second step, we need $\hat{P}_{S}^{*} / P_{S}=1 / \theta$ for all states having $\hat{P}_{S}^{*}(s)>0$.

Case when $\theta=1$ corresponds to $\hat{P}_{S}^{*}=P_{S}$. By law of large numbers, empirical distribution for each interval would be $P_{S}$ with high probability. Thus the non-causal nature of transmitter CSI is rendered useless in this case because only 1 subinterval (i.e. $q=1$ ) suffices per message interval.

Thus this coding scheme gives the following sufficient condition for the capacity per unit cost with causal or non-causal CSIT to be the same.

Theorem 4 Let $\mu$ denote $\inf _{s \in \mathcal{S}}\left\{\frac{P_{S}(s)}{\hat{P}_{S}^{*}(s)}\right\}$ for an optimum $\hat{P}_{S}^{*}$ that achieves the capacity per unit cost for non-causal CSIT in (23). For equivalence of capacity per unit cost with causal and non-causal CSIT, $\mu$ should either be arbitrarily small or be equal to 1. Moreover, for all states in support of $\hat{P}_{S}$ (i.e. states having $\hat{P}_{S}(s)>0$ ) should achieve ${ }^{9}$ the infimum $\mu=P_{S}(s) / \hat{P}_{S}(s)$.

If $\mu$ tends to zero, the divergence $D\left(\hat{P}_{S}^{*} \| P_{S}\right)$ should tend to infinity to satisfy the above condition. In other words, its arbitrarily rare to observe the source distribution where energy is transmitted. This is because (by Sanov's theorem) the larger $D\left(\hat{P}_{S}^{*} \| P_{S}\right)$ is, the rarer it is to have empirical distribution like $\hat{P}_{S}^{*}$ when actual state distribution is $P_{S}$.

Note that the wideband fading channel and the wideband writing on dirty paper [8] satisfy the above Lemma, which gaurantees that capacity per unit cost is the same with causal or non-causal CSIT. The fraction of states $\theta$ where energy was transmitted was arbitrarily small there. Thus the above Lemma explains some reasons for the equivalence of the capacity per unit cost with causal and non-causal CSI for those channels.

With this background, we revisit the capacity achieving scheme for the non-causal CSIT case. The state vector of each length $n$ subinterval can be viewed as a superstate

\footnotetext{
${ }^{9}$ If $\mu$ tends to 0 , this clause can be relaxed as long as $H_{b}(\mu) / \mu$ approaches $D\left(\hat{P}_{S}^{*}|| P_{S}\right)$.
} 
of cardinality $|\mathcal{S}|^{n}$. Now each message in the code corresponds to an interval consisting of $q$ super-symbols (or subintervals).

A subinterval of empirical distribution $\hat{P}_{S}$ corresponds to a superstate with probability $\exp \left(-n D\left(\hat{P}_{S}|| P_{S}\right)\right)$. Energy is only transmitted in these rare subintervals. Non-causal CSIT of a length $n$ subinterval in the original channel corresponds to causal CSIT in the super-channel. The idea of subintervals has thus converted the channel with non-causal CSIT to a channel with causal CSIT.

The causal CSIT channel (with superstates) has some arbitrarily rare superstates where energy is transmitted for achieving capacity per unit cost. Hence by Lemma 4, the capacity per unit cost for this super-channel is the same with causal or non-causal CSIT. Since non-causal CSIT for the super-channel also means non-causal CSIT for the original channel, the capacity per unit cost of the super-channel for causal CSIT equals the capacity per unit cost of the original channel for non-causal CSIT. Thus even if Lemma 4 is not satisfied for the original channel directly, the idea of subintervals achieves the noncausal capacity per unit cost by converting the original channel to a super-channel for which Lemma 4 is satisfied. This is achieved by providing arbitrarily rare (super)states for transmitting energy.

\section{Acknowledgements}

Thanks to Robert Gallager for suggesting a simple On-Off fading channel, which prompted the writing on fading paper scheme. Shashi Borade also acknowledges numerous insightful comments and suggestions by Ashish Khisti.

\section{Appendix: Proof of Converse of Theorem 3}

We use a technique similar to 9] [8], which adapts a converse for capacity to a converse for capacity per unit cost. For a code of length $l$, by Fano's inequality we know the following necessary condition for transmitting a message $m$ chosen uniformly out of $M$ possible messages with error probability smaller than $\epsilon$.

$$
(1-\epsilon) \ln M \leq I\left(m ; Y_{1}^{l}\right)+H_{b}(\epsilon)
$$

where $H_{b}(\cdot)$ denotes the entropy of a binary variable as a function of its probability of being 1 . From the converse for the capacity of a channel with causal transmitter CSI, we know that [1]

$$
I\left(m ; Y_{1}^{l}\right) \leq \sum_{i=1}^{l} I\left(U_{i} ; Y_{i}\right)
$$

where $I\left(U_{i} ; Y_{i}\right)$ denotes the mutual information between the state to input mapping $U_{i}$ and output $Y_{i}$. The mapping $U_{i}$ is considered as a random variable of cardinality $|\mathcal{X}|^{|\mathcal{S}|}$. The mutual information can be thought as the mutual information of a channel with input $U_{i}$ and output $Y_{i}$, where the output transition probability for input $u$ is given by $P_{Y \mid U=u}$ in (18).

Now we introduce a time-sharing random variable $Q$, which is independent of all other variables and is uniformly distributed over integers from 1 to $l$. This gives the following 
upper bound

$$
\begin{aligned}
\sum_{i=1}^{l} I\left(U_{i} ; Y_{i}\right) & =l I\left(U_{Q} ; Y_{Q} \mid Q\right) \\
& =l\left(I\left(U_{Q}, Q ; Y_{Q}\right)-I\left(Q ; Y_{Q}\right)\right) \\
& \leq l I\left(U_{Q}, Q ; Y_{Q}\right)
\end{aligned}
$$

Defining $U=\left(U_{Q}, Q\right)$ and defining $Y=Y_{Q}$, we get the upper bound on $(1-\epsilon) \ln M$ as $l I(U ; Y)+H_{b}(\epsilon)$.

Now assume a weaker average cost constraint instead of the per codeword cost constraint in (17) as follows

$$
\mathcal{E}\left[\sum_{i=1}^{l} b\left(X_{i}\right)\right] \leq \nu
$$

Using the time sharing variable and later replacing $X_{Q}$ by $X$ gives

$$
\nu \geq \mathcal{E}\left[\sum_{i=1}^{l} b\left(X_{i}\right)\right]=\sum_{i=1}^{l} \mathcal{E}\left[b\left(X_{Q}\right) \mid Q=i\right]=l \mathcal{E}\left[b\left(X_{Q}\right)\right]=l \mathcal{E}[b(X)]
$$

Combining this with the upper bound on $(1-\epsilon) \ln M$ gives

$$
\frac{\ln M}{\nu} \leq \frac{I(U ; Y)+H_{b}(\epsilon) / l}{(1-\epsilon) \mathcal{E}[b(X)]}
$$

As $\epsilon$ can be arbitrarily small and $l$ can be arbitrarily large, we get $\ln M / \nu \leq I(U ; Y) / \mathcal{E}[b(X)]$ as the necessary condition for arbitrarily small error probability on a code. Thus a code with arbitrarily small error probability $\epsilon$ must satisfy $\frac{\ln M}{\nu} \leq \frac{I(U ; Y)}{\mathcal{E}[b(X)]}$ for some choice of random variable $U$ (which denotes a mapping from states to inputs).

Now note that mutual information $I(U ; Y)$ can be written as

$$
\begin{aligned}
I(U ; Y) & =\sum_{u} P_{U}(u) D\left(P_{Y \mid U=u} \| P_{Y}\right) \\
& =\sum_{u} P_{U}(u) D\left(P_{Y \mid U=u} \| P_{Y \mid U=0}\right)-D\left(P_{Y \mid U=0}|| P_{Y}\right) \\
& \leq \sum_{u} P_{U}(u) D\left(P_{Y \mid U=u} \| P_{Y \mid U=0}\right)
\end{aligned}
$$

where $P_{Y \mid U=0}$ indicates the output distribution when the state to input mapping is identically zero i.e. when 0 input is transmitted for any state. Also note that the expected cost can be written as

$$
\mathcal{E}[b(X)]=\sum_{u} P_{U}(u) \mathcal{E}[b(X) \mid U=u]
$$

Combining this with (28) gives that any code with arbitrarily small error probability should satisfy

$$
\frac{\ln M}{\nu} \leq \frac{\sum_{u} P_{U}(u) D\left(P_{Y \mid U=u}|| P_{Y \mid U=0}\right)}{\sum_{u} P_{U}(u) \mathcal{E}[b(X) \mid U=u]}
$$

for some distribution $P_{U}(\cdot)$ of the state to input mapping $U$. In other words,

$$
\begin{aligned}
\frac{\ln M}{\nu} & \leq \sup _{P_{U}} \frac{\sum_{u} P_{U}(u) D\left(P_{Y \mid U=u} \| P_{Y \mid U=0}\right)}{\sum_{u} P_{U}(u) \mathcal{E}[b(X) \mid U=u]} \\
& =\sup _{u} \frac{D\left(P_{Y \mid U=u}|| P_{Y \mid U=0}\right)}{\mathcal{E}[b(X) \mid U=u]} \quad \text { Q.E.D. }
\end{aligned}
$$




\section{References}

[1] C. E. Shannon, "Channels With Side Information at the Transmitter," IBM Journal Research and Development, Vol. 2, pp. 289-293, 1958.

[2] S. Gel'fand and M. Pinsker, "Coding for Channel with Random Parameters," Problems of Control and Information Theory, Vol. 9, No. 1, pp. 19-31, 1980.

[3] C. Heegard and A. Gamal, "On the Capacity Computer Memory with Defects," IEEE Transactions on Information Theory, Vol. 29, No. 5, pp. 731-739, September 1983.

[4] M. Costa, "Writing on dirty paper," IEEE Transactions on Information Theory, vol. 29, no. 3, pp. 439-441, May 1983.

[5] R. Kennedy, Fading Dispersive Communication Channels, New York: WileyInterscience, 1969,

[6] I. E. Telatar and D. N. C. Tse, "Capacity and mutual information of wideband multipath fading channels," IEEE Trans. on Information Theory, vol. 46, pp. 13841400, July 2000.

[7] S. Borade and L. Zheng, "Wideband fading channels with feedback," Proc. Allerton Conference, Sept. 2004.

[8] T. Liu and P. Vishwanath, "Opportunistic orthogonal writing on dirty paper," submitted to IEEE Trans. on Information Theory.

[9] S. Verdu, "On channel capacity per unit cost," IEEE Transactions on Information Theory, Vol. 36, No. 5, pp. 1019-1030, September 1990.

[10] H. David, Order Statistics, 1st ed., New York: Wiley, 1970.

[11] P. Dupuis, R. Ellis, A Weak Convergence Approach to the Theory of Large Deviations, Wiley-Interscience, 1997.

[12] "Writing on dirty paper with causal CSI and effects of intereference tail" In preparation. 